\title{
A Result on Hamiltonian Line Graphs Involving Restrictions on Induced Subgraphs
}

FACULTY OF APPLIED MATHEMATICS

UNIVERSITY OF TWENTE

7500 AE ENSCHEDE

THE NETHERLANDS

\section{ABSTRACT}

It is shown that the existence of a Hamilton cycle in the line graph of a graph $G$ can be ensured by imposing certain restrictions on certain induced subgraphs of $G$. Thereby a number of known results on hamiltonian line graphs are improved, including the earliest results in terms of vertex degrees. One particular consequence is that every graph of diameter 2 and order at least 4 has a hamiltonian line graph.

\section{INTRODUCTION}

Let us call a condition on a graph $G$ with $n$ vertices numerical if it implies $\Delta(G) \geq \varepsilon n$ for some constant $\varepsilon>0$, where $\Delta(G)$ denotes the maximum degree of vertices of $G$. Several numerical and nonnumerical sufficient conditions on a graph $G$ ensuring that the line graph of $G$ is hamiltonian are known. Often a comparison between a numerical and a nonnumerical condition yields that there are many graphs satisfying the former but not the latter, and vice versa. Here we derive a nonnumerical condition that is weaker than several known conditions, including the earliest numerical ones. The condition involves certain restrictions on induced subgraphs isomorphic to one of the graphs in Figure 1.

\section{TERMINOLOGY AND PRELIMINARY RESULTS}

We use [2] for basic terminology and notation, except that we will speak of line graphs instead of edge graphs. We consider simple graphs only. Let $G$ be a 
graph. The line graph of $G$ is denoted $L(G) . G$ is pancyclic if $G$ contains a cycle of length $i$ for each $i$ with $3 \leq i \leq|V(G)|$. Like in [1], a nontrivial eulerian subgraph of $G$ will be called a circuit. Hence a subgraph $C$ of $G$ is a circuit if and only if $C$ is nontrivial and connected and every vertex of $C$ has even degree in $C$. In particular, if $C$ is a circuit, then $C$ is 2-edge-connected, and so is every block of $C$. A circuit $C$ is a dominating circuit or $D$-circuit of $G$ if every edge of $G$ is incident with at least one vertex of $C$. In proving the main result we will use the following well-known theorem:

Theorem 1 (Harary and Nash-Williams [9]). The line graph $L(G)$ of a graph $G$ is hamiltonian if and only if $G$ has a $D$-circuit or $G$ is isomorphic to $K_{1, s}$ for some $s \geq 3$.

If $C$ is a circuit and $Z$ a cycle of $G$ such that $V(Z) \cap V(C) \neq \emptyset \neq V(Z) \cap$ $(V(G)-V(C))$ and $G[E(C) \Delta E(Z)]$ (where $\Delta$ denotes symmetric difference) is connected, then $Z$ is called a $C$-augmenting cycle. Clearly, if $C$ is a circuit and $Z$ is a $C$-augmenting cycle, then $G[E(C) \Delta E(Z)]$ is also a circuit, and $|V(G[E(C) \Delta E(Z)])|>|V(C)|$, implying the following lemma.

Lemma 2. Let $G$ be a graph and $C$ a circuit of $G$ with maximum number of vertices. Then $G$ contains no $C$-augmenting cycle.

A special case of Lemma 2 is the following (cf. [1, Lemma 2]).

Lemma 3. Let $G$ be a graph and $C$ a circuit of $G$ with maximum number of vertices. Then $G$ contains no cycle $Z$ satisfying

$V(Z) \cap V(C) \neq \emptyset \neq V(Z) \cap(V(G)-V(C))$ and $|E(Z) \cap E(C)| \leq 1$

Another simple but useful observation is the following:

Lemma 4. Let $x_{1} x_{2} \ldots x_{r}$ be a path in a graph $G$ and let $i$ and $j$ with $1 \leq i<$ $j<r$ be integers such that $x_{i} x_{i+1}$ and $x_{j} x_{j+1}$ are edges of the same block $B$ of $G$. Then $x_{k} x_{k+1} \in E(B)$ for every integer $k$ satisfying $i<k<j$.

By $\tau$ and $\tau^{+}$we denote the graphs depicted in Figure 1. When one of these graphs is under consideration, the vertices will be referred to as $a, b, c, a_{1}, a_{2}$ like in Figure 1, unless names for these vertices have already been specified (e.g., when the graph occurs as subgraph of another graph).

$H_{m, n}$ denotes the graph obtained when a copy of $K_{m}$ and a copy of $K_{n}$, disjoint with the copy of $K_{m}$, are joined by exactly one edge. For $n \geq 8$, the graph $L_{n}$ is obtained by adding to a copy $H$ of $K_{n-6}$ the vertices $x, x_{1}, x_{2}, y, y_{1}, y_{2}$ and the edges $x x_{1}, x x_{2}, y y_{1}, y y_{2}, x_{1} y_{1}, x_{2} y_{2}, x u, y v$, where $u$ and $v$ are distinct vertices of $H$. 

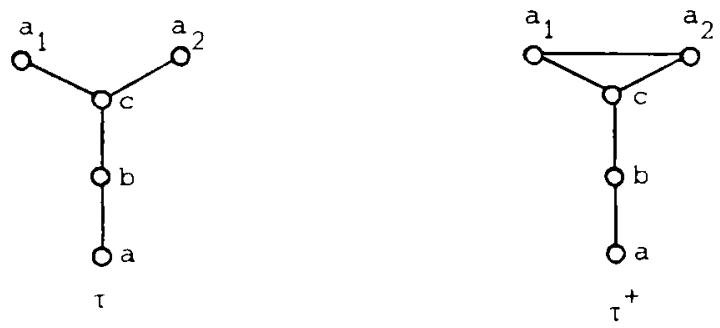

FIGURE 1.

\section{MAIN RESULT AND CONSEQUENCES}

Part of the arguments used to prove the main result below were also used in the proof of [1, Lemma 3].

Theorem 5. Let $G$ be a connected graph other than a tree, such that every induced subgraph isomorphic to $\tau$ or $\tau^{+}$with $d\left(a_{i}\right) \geq 2$ for $i \in\{1,2\}$ satisfies at least one of the following conditions:

(i) $|N(a) \cap N(c)| \geq 2$;

(ii) $|N(b) \cap N(c)| \geq 1$;

(iii) for $i=1$ or $i=2,\left|N(b) \cap N\left(a_{i}\right)\right| \geq\left\{\begin{array}{l}2 \text { if } a_{1} a_{2} \in E(G) \\ 3 \text { if } a_{1} a_{2} \notin E(G)\end{array}\right.$;

(iv) for $i=1$ or $i=2,\left|N(a) \cap N\left(a_{i}\right)\right| \geq 1$.

Then $L(G)$ is hamiltonian.

Proof. Suppose the hypothesis of the theorem holds, but $L(G)$ is nonhamiltonian. Let $C$ be a circuit of $G$ with maximum number of vertices. By Theorem $1, C$ is not a $D$-circuit of $G$, so there exists a path $u_{1} u_{2} v$ with $u_{1}, u_{2} \notin V(C)$ and $v \in V(C)$. Let $v v_{1}$ and $v v_{2}$ be two edges of $C$. From Lemma 3 we deduce that the subgraph $H_{1}$ of $G$ induced by $\left\{u_{1}, u_{2}, v, v_{1}, v_{2}\right\}$ is isomorphic to $\tau$ or $\tau^{\prime}$, while $\left(N\left(u_{1}\right) \cap N(v)\right)-\left\{u_{2}\right\}=N\left(u_{2}\right) \cap N(v)=\emptyset$. Observing that $d_{G}\left(v_{i}\right) \geq d_{C}\left(v_{i}\right) \geq 2(i=1,2)$, we conclude that $H_{1}$ satisfies (iii) or (iv).

Assume $H_{1}$ satisfies (iii). We distinguish two cases.

Case 1. $v_{1} v_{2} \in E(G)$.

Assume without loss of generality that $u_{2}$ and $v_{1}$ have a common neighbor $w$ with $w \neq v$. By Lemma 3, $v_{1} w \in E(C)$. If $v_{1} v_{2} \in E(C)$, then $v u_{2} w v_{1} v$ is a $C$-augmenting cycle, a contradiction with Lemma 2. If $v_{1} v_{2} \in E(G)-E(C)$, then $v u_{2} w v_{1} v_{2} v$ is a $C$-augmenting cycle, again a contradiction. 


\section{Case 2. $v_{1} v_{2} \notin E(G)$.}

Assume without loss of generality that $u_{2}$ and $v_{1}$ have two common neighbors $w_{1}$ and $w_{2}$ other than $v$. By Lemma 3, $v_{1} w_{1}$ and $v_{1} w_{2}$ are edges of $C$. If $v_{1} v$, $v_{1} w_{1}$ and $v_{1} w_{2}$ are in the same block of $C$, then $C-\left\{v_{1} w_{1}, v_{1} w_{2}\right\}$ is connected, implying that $u_{2} w_{1} v_{1} w_{2} u_{2}$ is a $C$-augmenting cycle, contradicting Lemma 2. If, for example, $v_{1} v$ and $v_{1} w_{1}$ are in different blocks of $C$, then $C-\left\{v_{1} v, v_{1} w_{1}\right\}$ is connected, since every block of $C$ is 2-edge-connected; $v u_{2} w_{1} v_{1} v$ is then a $C$ augmenting cycle, again a contradiction.

The contradictions in Case 1 and 2 show that $H_{1}$ satisfies (iv).

Let us call a path $P$ special if it satisfies the following requirements:

$-P$ has origin $v$

$-E(P) \subset E(C)$,

- each block of $C$ contains at most one edge of $P$, and

$-u_{1}$ and the terminus of $P$ have a common neighbor.

Note that, if $P$ is a special path, then, by the third requirement, $C-E(P)$ is connected.

Since $H_{1}$ satisfies (iv), $G$ contains a special path of length 1 . Let $P$ be a special path of maximum length, $x$ the terminus of $P, y$ the immediate predecessor of $x$ on $P$, and $z$ a common neighbor of $u_{1}$ and $x . z \notin V(P)$, otherwise $G$ contains the $C$-augmenting cycle $Q_{1} \cup v u_{2} u_{1} z$, where $Q_{1}$ denotes the $(v, z)$-subpath of $P$. Also, $z \neq u_{2}$, otherwise $P \cup v u_{2} x$ is a $C$-augmenting cycle. Furthermore, $x z$ is an edge of $C$, otherwise the cycle $Z_{1}$ with $Z_{1}=P \cup v u_{2} u_{1} z x$ is a $C$ augmenting cycle. Moreover, the edges $x y$ and $x z$ are in the same block of $C$; assuming the contrary, by Lemma 4, all edges of $E(P) \cup\{x z\}$ are in different blocks of $C$, again yielding the contradiction that $Z_{1}$ is a $C$-augmenting cycle. This contradiction is not obtained only if $\{x y, x z\}$ is a 2-edge cut of $C$. It follows that either $d_{C}(x)=2$ or $x$ is a cut vertex of $C$. If $d_{C}(x)=2$, then $G\left[E(C) \Delta E\left(Z_{1}\right)\right]$ consists of a trivial component and a component that is a circuit; the latter circuit contains one vertex more than $C$, contradicting the choice of $C$. Hence, in fact, $x$ is a cut vertex of $C$.

Let $B$ be a block of $C$ containing $x$ and different from the block that contains $x y$ (and $x z$ ). Then, by Lemma $4, B$ differs from all blocks of $C$ that contain an edge of $P$. Let $x x_{1}$ and $x x_{2}$ be two edges of $B$ and let $H_{2}=G\left[\left\{u_{1}, z, x, x_{1}, x_{2}\right\}\right]$. By Lemma 3, $u_{1} x \notin E(G)$. Also, $u_{1} x_{i} \notin E(G)$, otherwise $P \cup v u_{2} u_{1} x_{i} x$ is a $C$-augmenting cycle $(i=1,2)$. Since $P$ is a longest special path, $z x_{i} \notin E(G)$ ( $i=1,2)$. It follows that $H_{2}$ is isomorphic to $\tau$ or $\tau^{+}$. Since $d_{G}\left(x_{i}\right) \geq d_{C}\left(x_{i}\right) \geq$ $2(i=1,2), H_{2}$ satisfies (i), (ii), (iii), or (iv). The proof is now completed by deriving contradictions in all possible cases.

First suppose $H_{2}$ satisfies (i). Let $z_{1} \in\left(N\left(u_{1}\right) \cap N(x)\right)-\{z\}$. Like for $z$, we deduce that $z_{1} \notin V(P) \cup\left\{u_{2}\right\}, x z_{1}$ is an edge of $C$, and $x z_{1}$ and $x y$ are in the same block of $C$. Since $x z$ is also in this block, $C-(E(P) \cup\{x z\})$ is connected. But then the cycle $Z_{1}$ defined above is a $C$-augmenting cycle, a contradiction. 
Now suppose $H_{2}$ satisfies (ii). Let $y_{1} \in N(z) \cap N(x)$. By Lemma 3, $y_{1} \notin\left\{u_{1}, u_{2}\right\}$. If $y_{1} \in V(P)$, then $Q_{2} \cup v u_{2} u_{1} z y_{1}$, where $Q_{2}$ is the $\left(v, y_{1}\right)$-subpath of $P$, is a $C$-augmenting cycle, regardless of the question as to whether $z y_{1} \in E(C)$ or $z y_{1} \in E(G)-E(C)$. Hence assume $y_{1} \notin V(P)$. If both $x y_{1}$ and $z y_{1}$ are edges of $C$, then $Z_{1}$ is a $C$-augmenting cycle, while otherwise $P \cup$ $v u_{2} u_{1} z y_{1} x$ is, a contradiction.

Next suppose $\mathrm{H}_{2}$ satisfies (iii). Assume without loss of generality that

$$
\left|N(z) \cap N\left(x_{1}\right)\right| \geq \begin{cases}2 & \text { if } x_{1} x_{2} \in E(G) \\ 3 & \text { if } x_{1} x_{2} \notin E(G) .\end{cases}
$$

Let $x_{3} \in\left(N(z) \cap N\left(x_{1}\right)\right)-\{x\}$. Arguments used before show that $x_{3}$ cannot be a vertex in $V(P) \cup\left\{u_{1}, u_{2}\right\}$. If both $x_{1} x_{3}$ and $x_{3} z$ are edges of $C$, then $Z_{1}$ is a $C$ augmenting cycle. If both $x_{1} x_{3}$ and $x_{3} z$ are in $E(G)-E(C)$, then the cycle $Z_{2}$ with $Z_{2}=P \cup v u_{2} u_{1} z x_{3} x_{1} x$ is a $C$-augmenting cycle. Assume $x_{1} x_{3} \in E(G)-$ $E(C)$ and $x_{3} z \in E(C)$. By Lemma $4, x_{3} z$ is not an edge of the block $B$ of $C$ containing $x x_{1}$. As a consequence, $x_{3} z$ is not a cut edge of the connected subgraph $\left(C+x_{1} x_{3}\right)-\left(E(P) \cup\left\{x x_{1}\right\}\right)$ of $G$, since $z x \cup Q_{3} \cup x_{1} x_{3}$, where $Q_{3}$ is an $\left(x, x_{1}\right)$-path in $B-x x_{1}$, is a $\left(z, x_{3}\right)$-path in this subgraph. It follows that $Z_{2}$ again is a $C$-augmenting cycle. Now assume $x_{1} x_{3} \in E(C)$ and $x_{3} z \in E(G)-$ $E(C)$. We distinguish two cases.

\section{Case I. $x_{1} x_{2} \in E(G)$.}

Then $x_{3} \neq x_{2}$, otherwise $H_{2}$ would satisfy (ii). If $x_{1} x_{2} \in E(C)$, then $Z_{2}$ is a $C$-augmenting cycle. If $x_{1} x_{2} \in E(G)-E(C)$, then $P \cup v u_{2} u_{1} z x_{3} x_{1} x_{2} x$ is a $C$ augmenting cycle.

\section{Case II. $x_{1} x_{2} \notin E(G)$.}

Let $x_{4} \in\left(N(z) \cap N\left(x_{1}\right)\right)-\left\{x, x_{3}\right\}$. Like for $x_{3}$, we may assume $x_{4} \notin V(P) \cup$ $\left\{u_{1}, u_{2}\right\}, x_{1} x_{4} \in E(C)$ and $x_{4} z \in E(G)-E(C)$. If both $x_{1} x_{3}$ and $x_{1} x_{4}$ are edges of $B$, then $B-\left\{x x_{1}, x_{1} x_{3}\right\}$ is connected and hence $Z_{2}$ is a $C$-augmenting cycle. If, for example, $x_{1} x_{4} \notin E(B)$, then by Lemma 4 all edges of $E(P) \cup\left\{x x_{1}, x_{1} x_{4}\right\}$ are in different blocks of $C$, and hence $P \cup v u_{2} u_{1} z x_{4} x_{1} x$ is a $C$-augmenting cycle.

Finally, suppose $\mathrm{H}_{2}$ satisfies (iv). Assume without loss of generality that $N\left(u_{1}\right) \cap N\left(x_{1}\right) \neq \emptyset$. Then $P \cup x x_{1}$ is a special path longer than $P$, our final contradiction.

An immediate consequence of Theorem 5 is the following:

Corollary 6 (Oberly and Sumner [11]). If every edge of a nontrivial connected graph $G$ is contained in a triangle, then $L(G)$ is hamiltonian.

For other improvements of Corollary 6, we refer to [6]. 
The next result also is a trivial consequence of Theorem 5 .

Corollary 7. Let $G$ be a connected graph other than a tree. If $G$ contains no induced subgraph isomorphic to $\tau$ or $\tau^{+}$, then $L(G)$ is hamiltonian.

More general results on hamiltonian line graphs in terms of forbidden subgraphs can be found in [3].

Corollary 8. If $G$ is a graph of diameter at most 2 with $|V(G)| \geq 4$, then $L(G)$ is hamiltonian.

Proof. If $G$ has diameter 1 , then $G$ is complete, so $L(G)$ is hamiltonian by Theorem 1. If $G$ has diameter 2 , then every induced subgraph isomorphic to $\tau$ or $\tau^{+}$satisfies (iv) of Theorem 5. Hence either $L(G)$ is hamiltonian or $G$ is a tree. In the latter case, $G$ is isomorphic to $K_{1,|V(G)|-1}$ and $L(G)$ is hamiltonian by Theorem 1.

The graphs $H_{m, n}(m \geq 2, n \geq 2)$ and $L_{n}(n \geq 8)$ show that Corollary 8 is best possible, since they have diameter 3 and their line graphs are nonhamiltonian. Corollary 8 implies the following result, which was first proved (implicitly) by Lesniak-Foster and Williamson [10].

Corollary 9. Let $G$ be a graph with $|V(G)|=n \geq 4$. If, for every pair $u, v$ of nonadjacent vertices, $d(u)+d(v) \geq n-1$, then $L(G)$ is hamiltonian.

Proof. If the hypothesis holds, then every pair of nonadjacent vertices has a common neighbor, or equivalently, $G$ has diameter at most 2 . Hence Corollary 8 applies.

The graphs $H_{m, n}$ with $m \geq 2$ and $n \geq 2$ show that Corollary 9 is, in a sense, best possible.

Brualdi and Shanny [4] proved that $L(G)$ is hamiltonian if $G$ is a graph with $|V(G)|=n \geq 4$ and $|E(G)| \geq 1$ such that, for every edge $u v$ of $G, d(u)+$ $d(v) \geq n$. Clark [8] showed that for $n \geq 6$ the bound $n$ can be replaced by $n-1$ if $n$ is even, and by $n-2$ if $n$ is odd. An intermediate result is implied by Theorem 5 .

Corollary 10. Let $G$ be a graph with $|V(G)|=n \geq 4$ and $|E(G)| \geq 1$. If $G$ is not isomorphic to $P_{4}$ and, for every edge $u v$ of $G, d(u)+d(v) \geq n-1$, then $L(G)$ is hamiltonian.

Proof. Suppose $G$ satisfies the stated conditions. Clearly, $G$ has exactly one nontrivial component. Assume without loss of generality that $G$ is connected. If $G$ is isomorphic to $K_{1, n-1}$, then we are done. Otherwise, $G$ is not a tree. Let $H$ be an induced subgraph of $G$ isomorphic to $\tau$ or $\tau^{+}$. If 
$N(b) \cap N(c) \neq \emptyset$, then $H$ satisfies (ii) of Theorem 5, and we are done. Hence assume $N(b) \cap N(c)=\emptyset$, implying that $d(b)+d(c) \leq n$. If $d(b)+d(c)=$ $n-1$, then since $d(a)+d(b)+d(c)+d\left(a_{1}\right) \geq 2(n-1)$, we have $d(a)+d\left(a_{1}\right) \geq n-1$, implying that $H$ satisfies (iv) of Theorem 5 . Henceforth assume $d(b)+d(c)=n$, implying that $N(b) \cup N(c)=V(G)$. We distinguish two cases.

Case 1. $a_{1} a_{2} \notin E(G)$.

We have $d(a)+d(b)+d(c)+d\left(a_{1}\right) \geq 2(n-1)$, so $d(a)+d\left(a_{1}\right) \geq$ $n-2$. Since $a_{2} \notin N(a) \cup N\left(a_{1}\right), a$ and $a_{1}$ have a common neighbor, implying that $H$ satisfies (iv) of Theorem 5 .

Case 2. $a_{1} a_{2} \in E(G)$.

Then $a_{1}$ or $a_{2}, a_{1}$ say, has degree at least $\frac{1}{2}(n-1)$. If $d(b) \leq \frac{1}{2}(n-1)$, then $d(a) \geq \frac{1}{2}(n-1) \geq d(b)$, implying that $N(a)-N(b) \neq \emptyset$ and $H$ satisfies (ii) of Theorem 5. If $d(b) \geq \frac{1}{2} n$, then $d\left(a_{1}\right)+d(b) \geq n$, implying that $H$ satisfies (iii) of Theorem 5 .

If $n$ is even, then Corollary 10 is, in a sense, best possible, as can be seen by considering $H_{n / 2, n / 2}$ for $n \geq 6$. (Clark's result is also best possible if $n$ is odd, as shown by $H_{(n-1) / 2,(n+1) / 2}$ for $n \geq 7$.)

Numerical results in [1], [5], [7], and [12] show that the bounds in Corollaries 9 and 10 can be considerably decreased by imposing certain necessary conditions for hamiltonicity of $L(G)$, such as the nonexistence of a cut edge in $G$ incident with two vertices of degree at least 2 .

\section{CONCLUDING REMARKS}

Suppose we want to know whether a given graph $G$ with $n$ vertices satisfies the hypothesis of Theorem 5. At first sight it seems that $O\left(n^{5}\right)$ subgraphs isomorphic to $\tau$ or $\tau^{+}$have to be checked, since $\tau$ and $\tau^{+}$have five vertices. However, we can do better. The following algorithm, leaning on the proof of Theorem 5 , will give us either a $D$-circuit of $G$, and hence a Hamilton cycle of $L(G)$, or an induced subgraph of $G$ isomorphic to $\tau$ or $\tau^{+}$that satisfies none of the conditions (i)-(iv). We start from an arbitrary circuit $C$ of $G$. If $C$ is a $D$-circuit, then we are done. Otherwise, referring to the proof of Theorem 5 , by checking $i$ subgraphs isomorphic to $\tau$ or $\tau^{+}$we either find an induced one satisfying none of (i) through (iv), or a $C$-augmenting cycle, or a special path of length $i$. Since any special path has length smaller than $n$, less than $n$ checks suffice to find either an induced $\tau$ or $\tau^{+}$satisfying none of (i) through (iv) or a $C$-augmenting cycle, and hence a circuit $C^{\prime}$ with more vertices than $C$. If such a circuit $C^{\prime}$ is found, we repeat the above procedure with $C$ replaced by $C^{\prime}$. Clearly, after less 
than $n$ repetitions of the procedure, we have either found a $D$-circuit of $G$ or an induced $\tau$ or $\tau^{+}$satisfying none of (i) through (iv). The algorithm thus requires no more than $O\left(n^{2}\right)$ checks. In particular, it follows that the complexity of checking the hypothesis of Theorem 5 is no higher than the complexity of checking numerical conditions like those in Corollaries 9 and 10.

The hypotheses of Corollaries 6, 7, 9, and 10 admit a stronger conclusion than hamiltonicity of the line graph. If a graph $G$ other than a cycle satisfies the conditions of one of these corollaries, then $L(G)$ is, in fact, pancyclic (see [11], [3] and, for example, [1]). The hypothesis of Theorem 5 does not admit this stronger conclusion. The Petersen graph, for example, satisfies the hypothesis of Theorem 5 (even that of Corollary 8), but its line graph has no cycle of length 4. Also, if all blocks of a graph $G$ are isomorphic to $C_{4}$ and no pair of cut vertices of $G$ is adjacent, then $L(G)$ is hamiltonian by Theorem 5 , but $L(G)$ has no cycle of length $|V(L(G))|-1$.

\section{References}

[1] A. Benhocine, L. Clark, N. Köhler and H. J. Veldman, On circuits and pancyclic line graphs. J. Graph Theory 10 (1986) 411-425.

[2] J. A. Bondy and U. S. R. Murty, Graph Theory with Applications. Macmillan, London and Elsevier, New York (1976).

[3] H. J. Broersma, Subgraph conditions for dominating circuits in graphs and pancyclicity of line graphs. Ars Combinat. 23 (1987) 5-12.

[4] R. A. Brualdi and R. F. Shanny, Hamiltonian line graphs. J. Graph Theory 5 (1981) 307-314.

[5] P. A. Catlin, Contractions of graphs with no spanning eulerian subgraphs. Preprint (1986).

[6] P. A. Catlin, A reduction method to find spanning eulerian subgraphs. $J$. Graph Theory 12 (1988) 29-44.

[7] P. A. Catlin, Spanning eulerian subgraphs and matchings. Preprint (1986).

[8] L. Clark, On hamiltonian line graphs. J. Graph Theory 8 (1984) 303-307.

[9] F. Harary and C. St. J. A. Nash-Williams, On eulerian and hamiltonian graphs and line graphs. Can. Math. Bull. 8 (1965) 701-710.

[10] L. Lesniak-Foster and J. E. Williamson, On spanning and dominating circuits in graphs. Can. Math. Bull. 20 (1977) 215-220.

[11] D. J. Oberly and D. P. Sumner, Every connected, locally connected nontrivial graph with no induced claw is hamiltonian. J. Graph Theory 3 (1979) 351-356.

[12] H. J. Veldman, Existence of spanning and dominating trails and circuits. J. Graph Theory 10 (1986) 23-31. 